\title{
Sensemaking and Resilience for Human Resources in Crises: Lessons From Crisis-Purposed Organizations
}

\author{
Lauren Rich \\ University of West Florida \\ Steve LeMay \\ University of West Florida \\ Marilyn M. Helms \\ Dalton State University
}

\begin{abstract}
Reviewing the best practices in crisis-purposed organizations is useful to help organizations better react to crises. The purpose of this research is to use the COVID-19 pandemic to better understand sensemaking and resilience by carefully studying crisis-purposed organizations for insights. We find that labor market myopia interferes with an organization's crisis response. An organization may overcome labor market myopia by leveraging HR strategies that emphasize agility and resilience and by adapting to technological changes. We focus on military special operations as the primary example of crisis-purposed organizations, but also refer to wildfire fighters and other emergency responders. These organizations differ in how they recruit and qualify participants, but they all suggest approaches for organizations whose primary focus is something other than crises, but who will face crises in any event.
\end{abstract}

Keywords: crisis management, organizational resilience, sensemaking, HR strategy

\section{INTRODUCTION}

Sensemaking is most often needed when our understanding of the world becomes unintelligible in some way. (Ancona, 2012, p. 4)

The recent pandemic has made parts of our world unintelligible at times. COVID19, as a global and a national crisis, changed our views of the environment in which organizations function, especially in the labor markets and in how human resources management (HRM) should respond. Crisis-purposed organizations can serve as models for other organizations in how to prepare for and deal with crises, especially in the way they deal with organizational sensemaking.

Karl Weick, who developed the notion of organizational sensemaking, wrote about the value of organizational sensemaking during crises. "If we can understand the process of sensemaking during a crisis, then we can help people to prevent larger crises by smarter management of small crises" (Weick, 1988, p. 308). COVID19 challenged the sensemaking in many organizations because of its novelty and the 
uncertainty about its nature, its spread, and its virulence. But that is precisely where organizational sensemaking works, in the realm of the unknown, when organizations are confronted with adaptive problems rather than technical problems (Ancona, 2012). The lessons from crisis-purposed organizations are important because organizations face uncertainty even in the absence of global crisis (Weick, 1993).

Human rational decision-making is a hybrid of formal logic and ecological logic (Pinker, 2021). This hybrid rationality is the foundation of sensemaking. Sensemaking matches the perceived circumstances of the environment with the plausible identities that make up an organization. Sensemaking then determines which cues should be used to create the organization's map of resources and actions.

The COVID-19 crisis has magnified the problems of sensemaking in the specific ecology of factor markets, notably in labor markets. In this study, we compare crisis-purposed organizations to lean organizations, organizations whose outlook toward capacity limits their ability to respond to longer, broader crises. In many instances, lean organizations are either too lean, or not lean in the right ways (Ivanov, 2021). We compare these two types of organizations on sensemaking, agility, and resilience. Our approach is inferential, but that also fits with sensemaking as a guiding concept (Miner, 2006).

We base our arguments and propositions on the following premises: 1) the human element is key to resilience, agility, and the consequent adaptive capacity (Lengnick-Hall, Beck, \& Lengnick-Hall), 2) organizational sensemaking leads to policies and actions that may change as the mental maps of the environment change (Weick, 1993; Ancona, 2012), and 3) in keeping with Pinker (2021), we view human rationality as a hybrid system of propositional reasoning based on logic and pattern recognition. This last premise is important. "Because our propositional reasoning frees us from similarity and stereotypes, it enables the highest achievements of human rationality, such as science, morality, and law" (Pinker, 2021, p. 108).

This paper contributes to the literature in several ways. First, it examines some common HR practices in the context of crises. It compares crisis-focused organizations to lean organizations in routine operation by using publicly available sources to explain the differences in HRM tactics. This paper also extends the reach and definition of Labor Market Myopia (LMM) (Opengart, Ralston, \& LeMay, 2018) as a factor in the failure of some organizations to become resilient or agile. LMM disrupts sensemaking as both an organizational process and as a perspective on organizational functions. Finally, it highlights the need for organizational sensemaking in Weick's (1988) terms, especially during a crisis.

The analysis starts by describing labor market conditions, military special operations, and lean organizations. It then reviews the literature on sensemaking, Labor Market Rivalry and Labor Market Myopia, the importance of HRM in a crisis, resilience, and agility. It develops and uses the TDOR model-training, deployment, operation, and reset to help compare crisis-purposed organizations to lean organizations. It then closes with propositions on how firms should respond to disruptions like COVID-19, how they should use sensemaking in contested labor markets and how they can avoid blind spots, stereotypes, and myopia. Finally, it calls for future research in this crucial area.

\section{Sensemaking, HRM Tactics and Crisis-Purposed Organizations}

Special Operations Groups (SOGs) train, deploy, operate, and reset. They must constantly deal with the twin issues of fighting the current fight and preparing for the next one (Radzius, Pang, \& Gandy, 2013). SOGs constantly manage crises, but their approach works for conventional organizations as well. Only the proportions of TDOR need to change. It is a question of understanding the context.

First, crises represent change, but sudden change rather than evolutionary change. Second, conventional organizations routinely experience change, although not necessarily crises. Rarely do so many organizations experience crises simultaneously, as in the COVID-19 pandemic. The COVID-19 pandemic, with all its attendant evils, has provided a laboratory in which to examine labor markets and the HRM practices that interface with them. This includes recruitment, job analysis, training, performance evaluation, compensation, and more.

Human resources tactics including job extensions and job rotations are routine for SOG teams. Team members often train one another in their specialties, and while television and movie depictions exaggerate the level of expertise of individual operators, the idea that they are at least adequate at a wide range of tasks 
remains true. Put another way, James Bond is a fictional character, but he is also merely an exaggerated version of a composite of real people (Milton, 2016).

While some SOG teams are deployed, other teams are training, often employing job extension and job rotation. Many SOGs spend more time training than being deployed, and more time deployed than working on assigned operation (Radzius et al., 2013; Koerner \& Staller, 2021). Most organizations cannot do this, but that does not mean that lessons cannot be learned from what the SOGs, special warfare operators (SWOs), and other crisis-purposed military groups do. This leads to the development of Figure 1, a training, deployment, operations, and reset (TDOR) model for organizational analysis. The TDOR model contrasts resilient organizations, lean organizations, and finally crisis-purposed organizations.

\section{FIGURE 1 \\ TRAINING, DEPLOYMENT, OPERATIONS, AND RESET MODEL FOR ORGANIZATIONAL ANALYSIS}

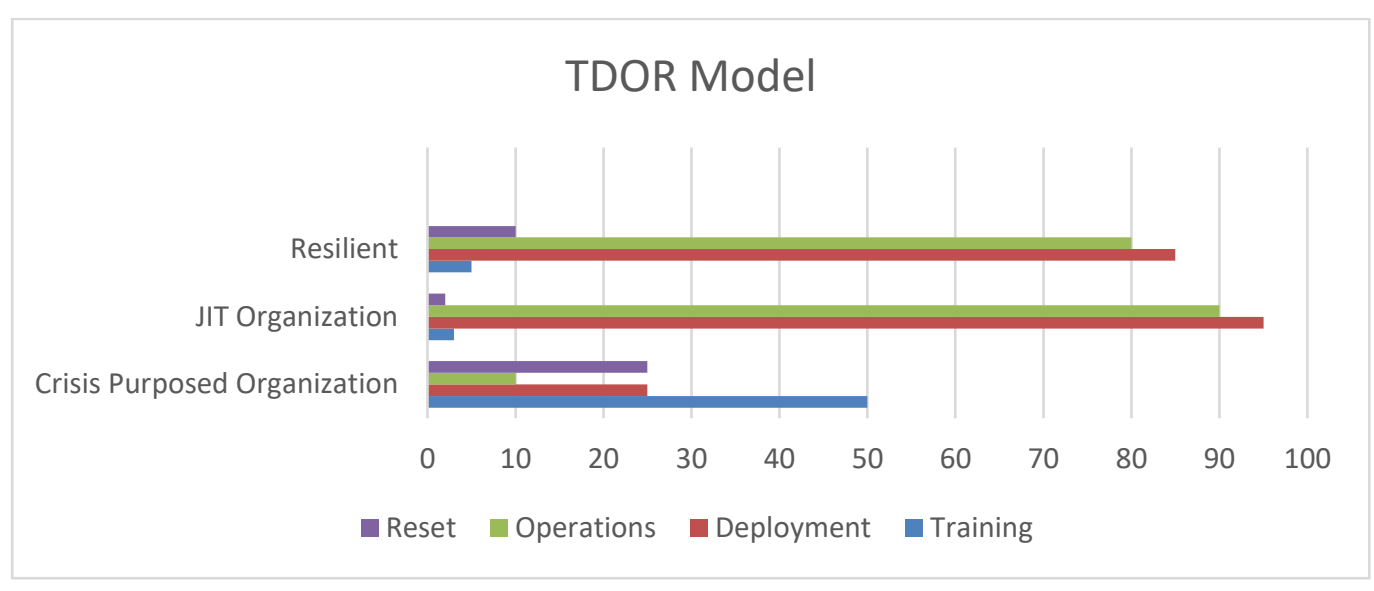

In the model, we distinguish between organizational resilience and organizational agility. First, a resilient organization is one that is prepared for crises. Resilience differs in that respect from an agile organization, which responds to crises only after the crises emerges or is identified. But resilience and agility operate together. Few organizations could be classified as resilient to every crisis, although military special operations may come the closest.

While organizational resilience and adaptation can be identified case-by-case and detail- by- detail, systemic antecedents like sensemaking may be less readily identified. Here, we contrast the crisis-ready, crisis-purposed military special operations groups with lean organizations, those that are inclined toward the concepts associated with JIT.

Most organizations have not reached full-blown just-in-time (JIT) or lean inventory practices, but it has conceptually influenced organizational strategy and behavior for decades since the strategy was popularized in the 1980's. The lean production movement, the lean organization, and lean processes all have merits: do as much as possible with as few resources as possible. However, following this guidance may mean a lack of resilience or agility. When a crisis arrives, the organization lacks necessary capacity to withstand the crisis or respond quickly.

COVID-19 magnified effects that always existed in labor markets but were ignored because the signals they gave to employers were too small to impel action. Here, organizational sensemaking failed in too many cases. These effects raise questions about how firms have responded and should respond not just to COVID19 disruptions in the labor market, but to other disruptions as well. The labor markets and human resources activities are laced with 'judgement calls,' but also afflicted by noise in making those judgements. Some of the noise comes from both LMM and LMR.

Lean organizations lack the capacity to fully execute sensemaking. While they may be able to enact cues and select cues, they cannot retain the maps the cues imply. They lack the capacity to develop agility 
or resilience because they fail to train or reset adequately. They simply do not allow slack for training and resetting. It does depend on how 'lean' is defined. Ivanov (2021) viewed lean organizations as potentially responsive to crises, notably the COVID19 pandemic, because they maintained what he termed 'resilient resources.' In the terms we use here, the lean organization has not made the adjustments that he recommended.

\section{LITERATURE REVIEW}

We start this literature review with sensemaking, then LMM and its antecedents, all of which can influence organizational sensemaking in the labor market. We also address the literature on the importance of HRM in crises.

\section{Organizational Sensemaking}

Organizational sensemaking is both a perspective and a process (Kudesia, 2017). In its narrow definition, it is a process where people "notice and interpret equivocal events and coordinate a response to clarify what such events mean" (Kudesia, 2017, p.1). Construed as a perspective, sensemaking develops a wholistic, recursive view of organizations and their interactions with their environments.

As defined and described by Weick, organizational sensemaking is improvisational. To follow the analogy, the environment sends messages to an organization, and the organization responds with "Yes, okay, and ...". What follows this "and" is the organization's effort to create order and make retrospective sense of events (Weick, 1993). This improvisation is grounded in identity construction and enacts sensible environments. It is an on-going, social process that focuses on cues extracted from the environment and is driven by plausibility rather than accuracy (Weick, 1995).

Miner (2006) described Weick's sensemaking as focused on the process of organizing, as centered around the rationality of small groups, as accepting of contradictory rationalities, as enacted by interdependent human actors, and as dedicated to removing uncertainty. Shared explanations for events allow organizations to function during times of great uncertainty. These shared explanations also enable collaboration (Van Epps, 2008).

FIGURE 2

\section{ORGANIZATIONAL SENSEMAKING PROCESS DIAGRAM}

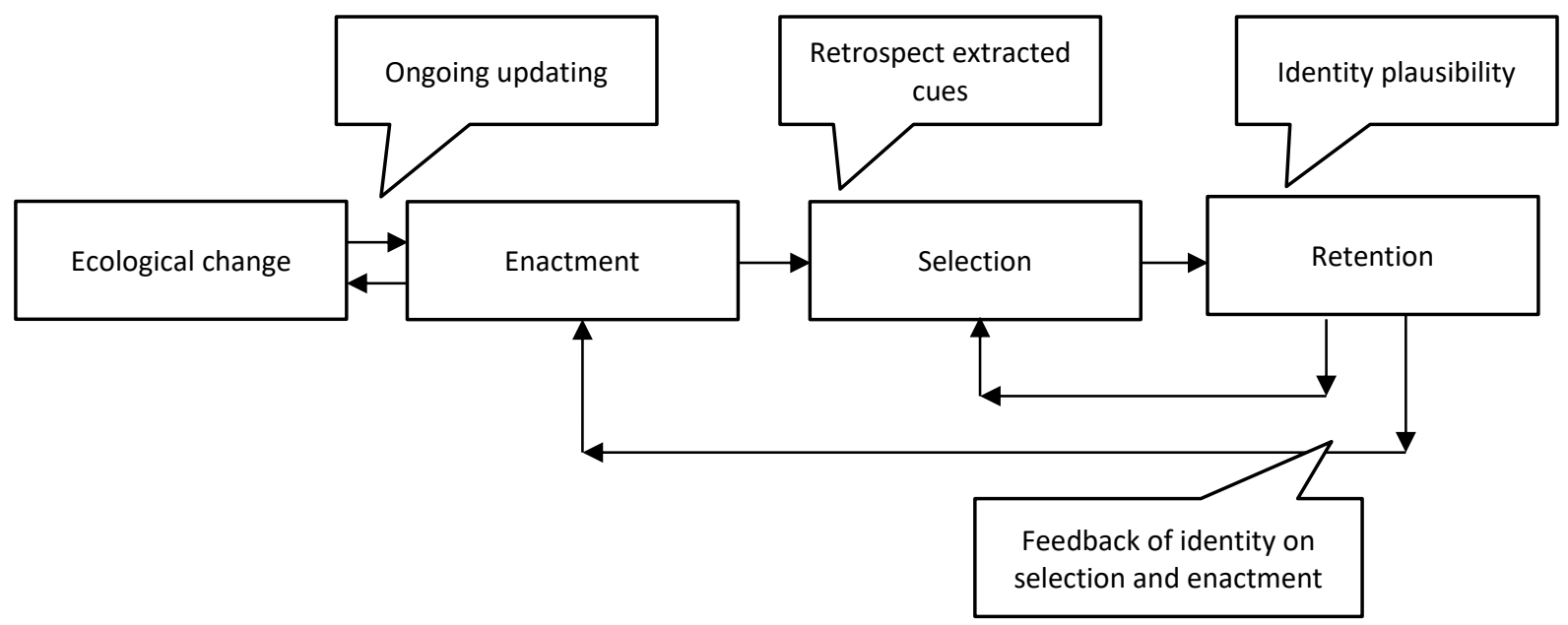

Weick, Sutcliffe, \& Obstfeld, 2005

Figure 2 shows Weick et al.'s (2005) visualization of sensemaking. Enactment involves noticing and identifying cues in the environment. Selection is choosing which of those cues is important. Retention 
involves determining whether the selected cues are plausible in terms of the self-identity of key individuals, the relational identity of individuals and groups, or the organizational identity. Organizations retain sets of cues, sometimes described as maps (Ancona, 2012), if they are plausible to the organization's sense of identity and if the feedback continues to support them.

Sensemaking collapses when feedback offers fundamental surprises or inconceivable events (Weick, 1993). That is when organizations need resilience most - to keep sensemaking from breaking down in the organization. Yet, as we argue here, sensemaking also contributes to organizational resilience. The two constructs interact with one another in ways that may benefit an organization or bring about its downfall.

Organizations have four sources of resilience that keep sensemaking from breaking down. First, improvisation and bricolage mean that organization can create order from the available resources. Second, organizations and key individuals can evaluate environmental maps virtually, in their heads, thus eliminating many choices before they reach the stages of enactment, selection, or retention. Third, organizations can adopt an attitude of wisdom, the idea that knowledge and ignorance grow together-the more we know, the more we know we don't know. Fourth, the interdependent individuals and groups interact respectfully, allowing for a synthesis of meaning from the joint subjectivity of individuals (Weick, 1993).

Much of the support for organizational sensemaking as a process and as a perspective comes from qualitative research partly because the concept is ambiguous, even amorphous, and so does not attract quantitative research (Miner, 2006). Weick (1988) studied two of the organizations that we examine here, the military and firefighters. The model that we developed, the Training-Deployment-Operations-Reset (TDOR) model, is discussed in another section.

Sensemaking offers a distinct perspective on what organizations are, how they function, and how they should be studied, especially in the context of a crisis (Kudesia, 2017). It should be clear what Weick himself found - that sensemaking is more about invention than discovery (Weick, 1995). Fundamental to the whole idea of sensemaking is this quote from Weick (2003, p. 186): "People create their own fate. Organizations enact their own environments."

\section{Factor Market Myopia}

Factor Market Rivalry (Markman, Gianiodis, and Buckholz, 2009) and Marketing Myopia (Levitt, 1960) entail overlooking key aspects of a firm's markets. Factor Market Rivalry points out that firms compete for the means of production in markets where the focal firm is a buyer, not a seller. Other buyers in those markets may not operate in the same selling markets as the focal firm. Even if the focal firm has a sound idea of its markets for sales, it may overlook competitors for key resources in factor markets. These markets are generally defined as markets for capital goods, land, and labor. Factory Market Rivalry exists even after the focal firm has recognized its competitors. Factor Market Rivalry can be global, regional, or local.

By contrast, Market Myopia disappears once reality has been acknowledged. Market Myopia means overlooking competitors in the markets for products and services. In situations in which the focal firm is the seller, market myopia overlooks other sellers. This stems primarily from a poor definition of the focal firm's markets. Levitt (1960) used railroads as an example in his original version of the classic article. In his view, railroads saw themselves as being in the railroad business when they were really in the transportation business. This myopic view caused them to overlook competition from other modes. Trucking took away high-value freight; water carriers took away low-valued freight; airlines took away mail and passengers. In each of these cases, railroad executives overlooked competitors in the market for transportation services (Levitt, 1960).

The idea of Factor Market Myopia was developed by combining the ideas associated with Factor Market Rivalry and Market Myopia. It is possible for a firm to be myopic in its factor markets, overlooking potential sources of necessary means of production for a variety of reasons, including ignoring competition or ignoring resources because of cognitive biases (Ralston, LeMay, \& Opengart, 2017). These ideas were further refined to focus on the factor market for labor. FMM disrupts organizational sensemaking in factor markets. 
Labor Market Rivalry and Labor Market Myopia are both subsets of Factor Market Rivalry and Factor Market Myopia (Opengart et al., 2018). Labor Market Rivalry focuses on the competition that organizations face involving human resources. Labor Market Myopia signifies poor judgements associated with how an organization defines its labor market and makes decisions that influence the supply and demand for labor sources. LMM may comprise stereotypes, false similarities, and biases, all of which run counter to rationality as defined by Pinker (2021). In our argument and in the model that we develop here, disrupted rationality means less effective sensemaking and consequent poor HRM strategies and tactics.

\section{COVID-19s Impacts on Labor Markets}

COVID-19 has highlighted the need for effective organizational sensemaking. The global health crisis associated with COVID-19 has caused an unprecedented level of disruption to labor markets around the world. COVID-19 has increased the skills gap and created significant career shocks for workers. As a result, organizations are struggling with decreased worker productivity, a shrinking workforce, and antiquated economic incentives (Agrawal, De Smet, Lacroix, \& Reich, 2020; Mullen, 2021). The term "The Great Resignation" has been used to describe events in the current labor market. People are quitting their jobs at record rates. Officials and researchers remain uncertain about explaining this phenomenon because it is early and because many potential explanations have offered themselves (Rosalsky, 2021; Cook, 2021). All of this reflects the kind of uncertainty that warrants active sensemaking.

\section{Skill Gaps}

Skill gaps have contributed to decreased productivity among existing workers. For years, rapid technological advances associated with artificial intelligence and automation, along with evolving consumer preferences and innovative business models, have contributed to the current gap in skills between the human capital demanded and human capital supplied. Yet, it seems to have taken a global pandemic to institute a sense of urgency within most companies worldwide to redesign jobs and rethink Human Resources strategies accordingly.

As human capital is redefined, many organizations are struggling to match existing employees to new roles and skills. Indeed, almost $87 \%$ of executives recently surveyed by McKinsey and Company voiced concerns about the skills gap in the workforce, but more than half of those concerned were not sure how to address the problem (Agrawal et al., 2020). Optimally, the existing skills gap would narrow as workers are gradually retrained and reskilled. However, recent circumstances have been far from optimal, and many organizations were not prepared to meet contemporary labor demands that were accelerated by COVID-19.

As a result, many organizations have responded to the skills gap with a short-term, almost survival-like focus. To absorb the new, in-demand skills, workers have been reshuffled into new positions through, job rotation, or given increased roles and responsibilities (i.e., job enlargement), but these actions are often implemented despite the lack of employee qualifications and without the proper training, resources, or support. While job rotation and job enlargement are not inherently negative HR practices, they both require substantial planning and training if they are to succeed. The short-term orientation reflects a long-term hesitancy to treat training and development as an investment rather than an expense (LeMay \& McMahon Jr., 1999).

\section{Shrinking Workforce and Antiquated Economic Incentives}

Akkermans, Seibert, and Mol (2018) identified career shock among workers who experience an exceptionally disruptive event that they cannot control. They often respond by spending considerable time deliberating over their career choices, commitments, priorities, and so on. The career shock fueled by COVID-19 has been no less than profound. Social distancing and remote working arrangements have compounded the effects by providing workers with optimal conditions to reflect on and reevaluate their personal and professional priorities.

This career shock has manifested itself in part as "The Great Resignation" (Hirsch, 2021). Even as the health risks associated with COVID-19 recede, the pandemic's effects on the labor market will continue. It will cripple organizations that lack resilient or rapid adaptive responses. The COVID-19 career shock has 
contributed to a shrinking workforce and may have radically altered the relationship between employers and employees, between organizations and their labor markets. Here, sensemaking still belongs to the organization and its leadership. Individual workers have only limited control over the actions that they can take, regardless of the quality of their sensemaking.

Some workers have found staying home to be preferable to going to work for personal or economic reasons. Some prefer remote work and others do not plan on returning to the workforce at all, remotely or otherwise. Other workers find themselves between jobs, lacking the qualifications for the jobs that are available in their area, lacking the means to move to where jobs are, or choosing to retire sooner than originally planned (AARP, 2021). The changing composition of labor demand has left other workers inbetween jobs and searching for positions that match their existing skills. While the latter scenario causes only temporary reductions to the workforce, government support has allowed workers to take longer to find new positions, so both scenarios reduce the number of available workers at any given time. Still, government support explains only a small portion of the change in the workforce composition. Researchers are still trying to understand the situation (Hirsh, 2021).

The career shock from COVID-19 has also significantly changed what employees expect from their employers. As a result, traditional economic incentives have become antiquated. The pandemic has significantly increased the amount of stress and anxiety for workers. In addition to the direct stressors induced by health concerns associated with COVID-19, many workers are facing challenging, ambiguous, conflicting, and often unreasonable job demands. In turn, the type and level of support expected by employees from their employers has evolved as well. Employees will likely require more emotional support from their employers in general, and organizations need to focus on the mental health and well-being of their employees.

\section{Resilience, Agility, and Adaptation}

Few organizations are only resilient or only agile. If we view resilience and agility as the consequences of sensemaking, we make the relationship between the concepts more readily understood. Resilience and agility both allow a firm to adapt. From the perspective of this research, resilience and adaptability differ in time-and adaptation can feature as an aspect of resilience or as an aspect of agility. Resilient organizations are prepared for the impact of crises and incorporate adaptive capacity; agile organizations adapt to crises as they arrive. We recognize that this is taking a portion of what has been written about defining resilience in organizations and relying only on these definitions. The study of organizational resilience is still in early development, so definitions of the terms proliferate. We are using ideas adapted from Hillman and Guenther (2021) in their analysis of definitions of resilience and their review of the literature.

Resilience is also a precursor to sensemaking, but in the context of sensemaking it can be both a product and a precursor. Weick (1993) mentioned the four sources of resilience discussed earlier as crucial to recovering from the loss of meaning that stems from the fundamental surprise or the inconceivable event. Crises like COVID-19 demand that organizations create order out of whatever is at hand, that they consider alternatives virtually, that they understand more and understand what they don't know, and that they collaborate - if they expect to thrive or even survive. But resilience as both a product and a precursor also add yet another dimension to the issue of definitions.

The same problem is true for definitions of agility (Walter, 2021). Agility is ill-defined and unlikely to be better defined given the approaches that the literature has taken to the issue. Most of the definitions have incorporated the outcomes--'thriving and surviving'--as part of the organizational characteristic, agility (Walter, 2021). This violates basic principles of definition as outlined by Aristotle, among others (Robinson, 1954; LeMay, Helms, Kimball, \& McMahon, 2017). Given these issues, we define the two terms based in part on these summaries of definitions.

We define resilience as the degree to which an organization is prepared for crises or dramatic change. We defined agility as the ability of an organization to respond quickly to crises or dramatic change. We include the broader term "dramatic change" because not all changes are crises. A firm could see a rapid rise in demand and sales. While this could be dramatic, we would be unlikely to call it a 'crisis.' The issue with 
defining agility or resilience comes to this: some resilient organizations still fail. Some agile organizations still fail. Put another way, researchers survey and interview the survivors and thrivers, not the failed organizations. Neither resilience nor agility guarantees surviving or thriving. Rather, these characteristics improve the odds for an organization. We argue that further work needs to be done on defining these terms, but find these definitions adequate for the purposes of this exploratory research paper. We address this issue again in the call for further research. Having said that, we now address some of the strategies that may lead to resilience and agility. A key element for both is sensemaking.

In the instances of both resilience and agility, firms can be either or both and still fail for reasons that have nothing to with either. The concepts should not be defined as comprehensive and tied to every dimension of an organization. Nonetheless, we argue that stronger sensemaking means greater resilience and greater agility.

\section{The Importance of HRM}

We start with the idea that organizations are systems composed of infrastructure, equipment, and people. To the extent that these can change, they are at the core of organizational sensemaking. How much capacity should you have? How much of that capacity is determined by each of these factors? Answers to these questions comprise the maps that might be considered and retained in sensemaking.

This may seem to be a limited view of an organization, but these are the areas that allow for resilience or agility. Infrastructure tends to be fixed for the near term. New buildings, new roads, new bridges, new ports--all tend to be limited for an immediate moment in crisis. An organization may come up with better ways to use infrastructure, but new capacity may be difficult to acquire.

Equipment tends to be more flexible, but as the supply chain issues associated with the lengthening of the COVID-19 crisis have shown, new equipment can be a problem. In many instances, new equipment may be meaningless without people to operate it. Neither infrastructure strategies nor equipment strategies should be dismissed as potential support for resilience or agility, but they are not the focus of this paper. An organization can create more human capacity by hiring more people, by making current employees more effective or efficient, by embracing technology, or some combinations of these. Sensemaking constantly turns over the maps of resource availability. As these maps change, so do HRM strategy and tactics.

To hire more people, an organization must win the rivalry for labor, labor market rivalry, in a contest with a wider range of potential employers. That means advertising for and interviewing qualified candidates; but organizations often advertise for higher qualifications than they need in hopes of getting 'bargains.' To make employees more effective and more efficient, organizations must train and to reset current employees for job extension or job rotation. They may improve employee performance by embracing the right technologies, a process that should involve current employees. For any of these strategies to work, however, an organization must overcome LMM. Each of the problems described here is based on failed sensemaking.

Yet, despite such challenging circumstances, some organizations are surviving - and even thriving. Opengart et al. (2018) suggested ways to address these issues in labor factor markets before COVID-19. Specifically, Opengart et al. (2018) suggested organizations can 1) increase workforce productivity by leveraging technology and culture; 2) expand the workforce by recruiting nontraditional workers; and 3) develop effective economic remedies by revamping traditional performance evaluations and compensation practices. To put theses into practice, an organization may need its sensemaking function to scan through many maps of the labor market before settling on one that is satisfactory. The TDOR Model and crisispurposed organizations can help to better understand these maps and choose among them.

\section{Increase Workforce Productivity by Leveraging Technology}

Crisis-purposed organizations typically welcome technological assistance. Their focus is on dealing with the crisis, not protecting jobs, careers, or profits. A special operator in the field will use whatever technology helps get the job done. One of the authors tested equipment designed to increase endurance in the field for military personnel on foot. It required customization and could be deployed only by the 
individual involved, but it would increase efficiency and reduce the potential for injury and fatigue. There was no question that it would be readily accepted by field operators if it could be deployed. If it also means that fewer people are at risk, even better. The same attitude applies to fire fighters in the wildfires in California. These are people and organizations that would prefer to put themselves out of jobs.

That does not address the fear that other workers have of being displaced by technology. While leveraging technology fits easily into organizational sensemaking for crisis-purposed organizations, it does not fit so easily into organizations with ordinary purposes, even during a crisis. For some workers, the crisis will go away, but they wonder if their jobs will go away as well. Implementing such technology can be challenging. Associating technology with job replacement creates resistance. Apprehension surrounding certain technologies is valid, since technology has reduced the need for the human component of many occupations including travel agents, file clerks, and cashiers.

Nevertheless, technology does not always replace jobs. Sometimes, technology saves jobs (Opengart et al., 2018). In the wake of COVID-19, for instance, technology has enabled workers to remain productive despite enormous physical barriers. Workers will be less resistant when they see technology as a tool for success rather than a source of competition. For example, routine medical appointments may be conducted online, including follow up visits after surgery and visits to communicate test results, renew prescriptions, and so on. These online telemedicine visits are likely to remain in the stock of services offered by medical service providers.

Some organizations were already developing remote jobs, so they were prepared for COVID-19. COVID-19 also forced many organizations into remote work even though they were not fully prepared because they lacked the necessary infrastructure. For many organizations, even as the health risks of the pandemic begin to diminish, remote work remained. Many organizations have moved more rapidly into the world of remote work than they might have without the impetus of the pandemic. Other organizations are insisting on full return to in-person work. It seems these organizations are having a harder time embracing technological change than the workers they employ, or once employed. Most organizations need to be fast tracking the technology that makes workers productive and effective from home. In addition to focusing on the necessary technologies, companies must reevaluate their informal systems to ensure that their culture supports remote work, and that remote work reinforces their culture.

\section{Recruiting Nontraditional Workers}

While the career shock from COVID-19 caused some workers to exit the workforce altogether, the increased flexibility associated with remote work has simultaneously created opportunities for others to enter the workforce. Indeed, "the work reality fostered by the COVID-19 pandemic has helped to supersede structural, geographic, and social barriers that previously limited talent management decisions" (Caligiuri, De Cieri, Minbaeva, Verbeke, \& Zimmermann, 2020, p.705).

These new working conditions, which are likely to stick in many instances, open doors for more than just individuals needing flexibility. Introverts have become more comfortable suggesting ideas, providing constructive feedback, brainstorming, and communicating with their remote colleagues (Caligiuri et al., 2020). Extraverts often have an advantage through traditional recruitment and selection practices, but in the COVID-19 induced virtual world, the advantage seems to diminish (Wilmot, Wanberg, KammeyerMueller, \& Ones, 2019).

Recruiting and hiring practices must change before organizations can benefit fully from the potential of introverted candidates. Still, organizations can expand their workforce by recruiting non-traditional workers like those who need flexibility and the introverts. We should note that overlooking these candidates is a form of labor market myopia. It is also becoming increasingly clear that compensation and benefits packages and methods for evaluation need revision, although the nature of the revision is unclear and still not the subject of extensive research.

A number of companies are finding the pandemic offers the right time to adjust the evaluations process to meet those interests. More frequent, informal check-ins have become popular, in some cases because annual evaluations were too time-consuming and 
in-the-moment feedback can be more beneficial. Some leaders have already said such changes prompted by the pandemic will become permanent (Mullen, 2021, p.3).

As demonstrated here, the HR strategies developed by Opengart and colleagues in 2018 remain current despite the labor market disruptions onset by COVID-19. While every organization has been significantly impacted by COVID-19, each firm's experience has been unique. While many businesses have been forced to shut down or are still struggling to make ends meet, other businesses have demonstrated they can not only survive COVID-19 but thrive under the same conditions as their failing competitors.

Each aspect of these HRM strategies, tactics, and constructs depends on sensemaking or affects sensemaking in some way. As described in Table 1, these ideas either affect sensemaking, inspired the need for sensemaking, or exemplify the outcomes of sensemaking. Base on these ideas and circumstances, we now resort to our own versions of proposition development as described by Pinker (2021).

\section{PROPOSITIONS}

This literature review and environmental analysis leads to the following propositions. We summarize the propositions with a more focused model of organizational sensing in the labor market.

Proposition 1: LMM interferes with organizational responses to crises like COVID-19 by disrupting sensemaking.

Labor market myopia, LMM, like all forms of myopia, is a lack of imagination, intellectual insight, or more likely, in the case of the COVID-19 pandemic, a lack of foresight. All of these may be improved through better sensemaking. Today's workforce and management alike have experienced few if any pandemics or labor pressures except the ebbs and flows of the economy, with its attendant unemployment, but nothing to the scale of COVID.

In the absence of effective sensemaking and the foresight it provides, few employers were prepared to deal with shortages, shutdowns, and quarantine. We quickly learned that employees, post-COVID, have come to value flexibility, control over work hours and work delivery, often favoring work-from-home options that allow for childcare and homecare tasks to be completed often simultaneously. Employers who did not prepare for pandemic events struggled. Many had not supported remote work, yet research shows that productivity was the same and, in some cases, even higher, when employees were allowed to work remotely and had the appropriate computer, printer, and supplies (Maurer, 2020). Companies with strong work-from-home programs were more resilient and had less myopia than counterparts.

From the perspective of sensemaking, many organizations struggled and still struggle to create plausible identities that accept the new conditions in the workforce. Organizations whose sensemaking fails them will most likely struggle in contested labor markets, markets with high levels of factor market rivalry.

Proposition 2: Organizational capacity to operate depends heavily on human resources capacity and strategy, where the quality of these factors depends in turn on sensemaking.

COVID-19 has brought huge and likely permanent changes to the labor market. While every organization has been significantly impacted by the global pandemic, each firm's experience has been unique. While many businesses have recently been forced to shut down or are still struggling to make ends meet, other businesses are not just surviving COVID-19 but thriving under the same conditions as their failing rivals. Thus, in a context of constant change, an organization's capacity to operate depends heavily on its ability to adapt. While adaptation has always been considered a valuable organizational capability, COVID-19 has demonstrated that being adaptive is no longer a viable source of competitive advantage but rather a criterion for survival. Simply put tomorrow's organizations will be the ones that successfully adapt to yesterday's changes. During the COVID-19 "great resignation", organizations are looking to human resources for answers. And without sufficient human resources, it is challenging to prepare for crises. 
While most organizations lack sufficient resources to prepare for crises like crisis-purposed organizations, we believe there are significant opportunities for improvement. We developed the TDOR model to extend the procedures commonly attributed to crisis-purposed organizations (i.e., training, deployment, operating, and resetting) to specific HR system components that can be leveraged to maximize any organization's capacity for resilience. While the TDOR model uses some terminology that is often associated with crises-purposed military organizations (e.g., deployment), notably, each step of the TDOR model mirrors an existing, core HR function; to this point, HR is responsible for training (corresponding to $\mathrm{T}$ ), job analysis (corresponding to $\mathrm{D}$ ), compensation planning (corresponding to $\mathrm{O}$ ), and performance evaluations (corresponding to R). We should also emphasize that the TDOR model represents a continuous process rather than a four-step sequence; thus, an organization's capacity for resilience increases incrementally with each completed cycle, not with each completed step.

While we propose that the TDOR model is generalizable in the sense that it can be applied to any organization that engages in training, job analysis, compensation planning, and performance evaluations, we must also recognize a set of challenges that threaten the effectiveness of TDOR, within an organizational context, if left unaddressed. Specifically, organizations must address sources of LMM before implementing the TDOR model. For years, organizations have overlooked the impact of technology on human capital and failed to recognize a strategic relationship between business operations and human resources in general. Both cognitive biases represent major sources of LMM that significantly interfere with TDOR.

Many contemporary organizations are trying to operate with HR systems that are essentially decades behind other business functions/systems. For years, rapid and disruptive advances in technology have contributed to an increasing gap between the human capital skills that organizations demand, and workers can supply, and now the pandemic has accelerated the urgency for organizations to close this gap at an unprecedented, aggressive pace. As a result, the pandemic has left many organizations struggling to understand how/why their HR systems did not evolve alongside the rest of the organization. Human resources functions were not created to be flexible.

Traditionally, HR functions were designed to respond to the needs of organizations (e.g., filling job vacancies, developing training when deficiencies are identified, terminating employees when employment contracts are broken, and solving employee-related problems). Further, many organizations did not consider human resources as a strategic function, at least not until COVID. As a result, critical HR functions (i.e., training, job-analysis, compensation planning, and performance evaluations) have evolved very slow and independently into a rigid, disconnected, antiquated systems. In short, the TDOR model will not contribute to organizational resilience unless rigid, traditional HR functions are overhauled/updated to match the flexibility and technological capabilities that's commonly observed through innovative business models and the accelerated pace of organizational change in general.

The "great resignation" provides a more tangible example of the ill effect associated with the all too common, antiquated HR practices; Organizations simply cannot train, deploy, operationalize, and reset quick enough to meet the current needs of their employees, so workers are just walking out. Such high levels of turnover also highlight another common issue that may be working against the TDOR model. Recall that developing a capacity for resilience, requires organizations to complete multiple TDOR cycles/passes. Following this line of reasoning, resetting may be the most critical TDOR cycle/phase. The reset phase requires organizations to not only evaluate/reflect on their performance but also grow from that evaluation/reflection; yet, growth is difficult, if not impossible, when employees are turning over faster than organizations can capture and incorporate their experiences into the next loop. In short, the TDOR model will not contribute to organizational resilience if turnover prevents or limits an organization's ability to reset.

Notably, organizations that have successful avoided LMM and excessive turnover-even despite the pandemic - seem to share a common characteristic: many of the organizations that are currently thriving invested heavily in HR technology, which allowed the HR functions most central to resilience (i.e., training, deploying/job analysis, operationalize/compensation management, and resetting/performance evaluation) to develop alongside business innovations and other support functions such as sales, accounting, marketing, etc. 
Companies like Fuel50, Gloat, and Hitch are just three of the many HR consulting firms that are shaking up the HR industry by developing internal talent marketplace platforms for a growing list of high-profile clients around the world. While the recent explosion of HR technology was spurred by COVID-19, it turns out that many high-profile organizations jumped onto this bandwagon long before COVID-19. For instance, Citi hired Fuel50 to develop its internal talent marketplace in 2011, with additional organizations such as eBay, Ericsson, Indeed, Celgene, RTI International, Vanguard, Unilever, Seagate, Carmax, Levi Strauss, and Patagonia following suit prior to 2019.

With the help of HR technology, organizations can develop an internal talent marketplace which is likely to leverage the benefits associated with a gig economy without having to compete externally for labor. The use of HR-related technology seems to have provided companies with an advantage at minimizing the barriers associated with LMM and turnover-ultimately making the TDOR process more effective. Specifically, organizations such as eBay, Ericsson, and Indeed have utilized HR technology to significantly increase the speed and flexibility of training programs, increase employee retention by emphasizing internal mobility, mobilize talent and work, identify more useful dimensions for matching employees with compatible work opportunities, make work portable, reduce discriminatory biases, customize incentives, and streamline performance reviews, just to name a few. Internal talent marketplaces not only support the TDOR model but also provide a catalyst for developing HR strategies, principles, and policies that promote organizational agility and resilience.

\section{HR Strategies for Agility}

Agile organizations can adapt quickly to change. Notably, people are the most flexible and versatile component of an organizational system, so transforming human capital is often the most immediate way for organizations to adapt when changes arise. Opengart and colleagues developed a set of HR strategies in 2018 that remains current in spite of the labor market disruptions onset by COVID-19. Specifically, organizations can 1) increase workforce productivity by leveraging technology and culture; 2) expand the workforce by recruiting nontraditional workers; and 3) develop effective economic remedies by revamping traditional performance evaluations and compensation practices. We propose that these three HR strategies will contribute to organizational agility when executed in a timely manner following an inflection point.

\section{HR Culture for Resilience}

COVID-19 highlighted that crisis management should not start at the time of crises; in other words, while agility is necessary for adaptation it is rarely sufficient for survival. As Peter Drucker once said, "culture eats strategy for breakfast". Applying this hyperbole, we propose that an organization's capacity for agility comes from its culture. Specifically, we synthesize research from Lengnick-Hall et al. (2011) to highlight key HR principles and policies that we propose will prepare an organization to effectively execute the tactical strategies discussed above. Developing a culture of resilience will maximize an organization's capacity to not only navigate the day-to-day turbulences but also prepare for crises to come.

Focusing on the contextual dimension of Lengnick-Hall et al.'s (2011) framework, specific HR principles and policies can help develop a capacity for resilience in several key areas. HR policies should encourage joint employee teams and networks, empowerment, open communication, results-based appraisals, and user-friendly integrated information systems. Likewise, HR principles should foster social interactions, broad resource networks, reciprocal trust and interdependence, facilitative communication, self-management/leadership, organizational orientation, personal accountability, power based on expertise (rather than hierarchical position), and recognition for contributions and/or outcomes (rather than tasks).

Proposition 3: Organizations that succeed in an LMR environment invest in training as part of their crisis response, and the nature of that training is determined by sensemaking.

Most organizations were unprepared for the COVID-19 pandemic and its effects on employees and labor sufficiency. However, lessons from the past can remind us of the benefits of cross training and training investment to offer the agility, flexibility, and ability to pivot in the time of crises. One example is from the 
hotel/motel industry. Hotels have long benefitted from cross-training employees, especially at smaller hotels where employees can work different jobs within the hotel including housekeeping, laundry, or restaurant. With cross-training, fewer employees are needed full time, resulting in overhead cost savings and profitability. Other benefits are staff with a strong competency and a sensitivity of the various job requirements. Employees may benefit from career advancement as they learn new roles. Many hotels use certification testing to ensure employees understand the new roles (Arlotta, 2018). Standardizing uniforms also sends a consistent message to customers who don't differentiate between employees and their jobs based on attire and helps to solidify the cross-training culture. During COVID-19, the travel industry, particularly hotels without such flexibility were forced to reduce services including catering and daily housekeeping and room cleanings due to a lack of workers skilled in multiple jobs. Others increased costs for the various amenities that were previously included in the nightly room costs to cover higher labor costs and cope with labor shortages.

Tekin, Hopp, and Van Oyen (2002) developed an Agile Workforce Evaluation framework to implement workforce agility. In one of their industry examples, Continental Plastic Containers had focused on training for machine operators but had not trained machine operators to troubleshoot production problems. Management created a career path for cross-training in a scheduled rotation to provide operator's additional experience. With more responsibility and better pay, operator retention increased and could dynamically address problems, reducing yield losses. Junior and Saltorato (2021) in their research on workforce agility found the most agile workforce is comprised of four interrelated, interdependent dimensions of proactivity, flexibility and adaptability, resilience, and competence. They found the best ways to promote agility is through learning and training, the forms of work organization, human resource management, and culture/organizational structure.

Asking teams to define flexibility is critical. As an example, at mining, metals and petroleum company BHP, a crew in Western Australia allow trucks to remain operational during scheduled meal breaks. The "bamboo crew," like bamboo, is strong and flexible, but firmly rooted (Gross, Morphet, and Lyons, 2020). Healthcare providers, based on their community service mission, often design their facilities and often their employees to be flexible in a crisis. For example, Vanderbilt University Medical Center in Nashville, Tennessee set up an overflow of beds set up in an adjacent parking garage built especially for such crises leading to flexibility in a pandemic (Hartnett, 2020). Having the garage space ready for such purposes provides flexibility for patient surges. Other hospitals have reported using office staff to help on patient floors in peak demand. While not trained as healthcare professionals, office staff can easily pivot to assist with data entry, paperwork, and even patient-facing tasks including delivering water and ice and fresh linens and towel along with bussing food trays.

In these cases, organizations showed agility in infrastructure as well as in human resources, but it was people who thought up the new ways to use infrastructure. These organizations came up with new ways to train, deploy, and reset their workforces. In each case, they used organizational sensemaking to arrive at plausible solutions that fit their identities as employers, healthcare organizations, or manufacturers.

\section{Proposition 4: If an organization is to be resilient, then it must overcome the effects of LMM.}

Perhaps the classic examples of LMM involve job and career stereotypes. LMM manifests itself through stereotypes of job suitability based on age, gender, race, and other characteristics. For example, Cheung \& Woo (2021) found that hotel managers thought older workers unsuited for customer contact jobs because they do not smile as much as younger workers. These same managers also thought that older workers were unsuited for jobs behind the hotel desk or waiting tables because they were too physically weak, but thought older workers to be suitable for housekeeping, a job that is at least equally demanding physically.

Job stereotypes based on gender have a long, sordid history. In a mixed methods study, Tang et al. (2017) found that not only do job listings contain heavily gendered language, but potential job applicants also carry gendered stereotypes in reading — often misreading - job postings. These included the idea that women were unsuitable for technical jobs, although the respondents saw customer service representative 
for cable services as a technical job. Several analyses even suggest that the stereotype that women are unsuited to technical jobs affects the market for economics faculty (Wu, 2017, 2018). González, Cortina, and Rodriguez (2019) conducted a field experiment using paired fictitious resumes. In the main experiment, the resumes changed only the gendered names of the applicants. The resumes were sent to 1372 job advertisements. They found biases that favored men over women, although bias was reduced when women had better qualifications. They conducted a second trial in which the resume signaled having children. Then, the bias against women rose. They attributed these biases to stereotypes in the minds of hiring officials (González et al., 2019).

In a landmark study on the history of gender stereotypes, Eagly, Nater, Miller, Kaufman, and Sczesny (2020) examined national polling of US citizens on gender stereotypes for 1946-2018. They found that gender stereotypes had indeed changed, but not in the way or to the degree often assumed in the U.S. They examined three areas: 1) communion, as in affectionate and emotional; 2) agency, as in ambitious and courageous; and 3) competence, as in intelligent and creative. They found that contemporary stereotypes convey substantial female advantage in communion, a smaller male advantage in agency, and gender equality in competence, with some hints of a female advantage in competence.

Stereotypes contribute strongly to LMM, but they are not the only factors involved in it. For example, there are strategic blind spots (LeMay, McMahon, Batchelor, \& Keller, 2020). Strategic blind spots overlap with stereotypes, but not all blind spots LMM involve stereotypes. At times, hiring officials and recruiters overlook a person or group of people because they have always looked elsewhere, because their communications channels do not reach the overlooked group, or because they know nothing about the group. Blind spots can also arise because of flaws in the analytical approach to the labor market (Zahra \& Chapels, 1993).

All these contributors to LMM also interfere with success in the labor market and in managing human resources. The human element of an organization is its most important source of resilience. No organization can respond readily to threats or disruptions without people who adapt quickly and appropriately. Take two key organizational responses to labor shortages or disruptions: job extension and job rotation. If companies fail to train people to perform the new duties associated with each of these tactics, then they are likely to create excess job stress for the employee, find that the employee performs poorly, or that the employee chooses to work elsewhere. This last is especially likely in conditions of LMR.

To create a more resilient workforce, firms should engage in job extension and job rotation on a more consistent basis, thus preparing the workforce for the possibility of disruption. Such training investments also send signals to the employees that they are valued, usually knowing that the training is expensive and that it increases their value not only to the company they now work for, but to the labor market. In this way, a firm can avoid LMM by avoiding the labor market entirely. Keep the workers you have, rather than fight for new workers. Human resources may elicit more competition-factor market rivalry--than other factors in supply chains (Balmer, 2011; Ellram, Tate, \& Feitzinger, 2013). While LMR may be unavoidable, avoiding LMM should be strategic priority for human resources.

Navy Federal Credit Union, for example, employs some 8,400 people in Escambia County, Florida, making it the county's largest employer. Even before the pandemic, the firm invested heavily in its employees, but the crisis demonstrated the firm's resilience. They recently increased the size of their campus to accommodate 10,000 employees but authorized a redesign as a result of recognizing the impact of the impact on their employees. They anticipate continuing with more remote work. The interior of the new building was altered in light of these circumstances. Beyond that, the organization offers employees consistent training programs, ready access to university programs, and continuous professional development. Their training department has six classrooms that remain busy most of the day and into the evening, offering certification programs and other career development opportunities. All of these amount to human resources responses to changes in strategy and circumstance.

LMM interferes with organizational sensemaking. That means not only does it interfere with the organization's interactions with the labor market, it interferes with the organization's internal workings and identities. 
Proposition 5: LMM leads not only to overlooking potential sources of new people, but also to overlooking opportunities to train existing employees to make the organization more resilient.

When Special Operations Groups qualify people to join their number, they need not recruit. Members of other parts of the organizations line up to attempt the brutal process of qualifying for membership in these elite groups. The trainers and leaders of these groups know that the applicants all have basic military skills in the same sense that professional sports teams rarely recruit people who have never played their game. An applicant for SOG status can already use a variety of weapons, just as a draftee for the National Basketball Association can already dribble. Membership in these organizations is sought, so recruiting is not the problem.

Still, even these organizations can suffer from LMM. A potential draftee might be considered too short to play in the NBA and a potential SOG member might be regarded as unqualified for specific tasks based on criteria that have little to do with the tasks at hand. Until an organization undertakes to train people in specific skills, its leaders cannot know the capabilities of its workforce.

Most organizations find themselves in positions that differ from the two examples just discussed. They must recruit candidates or decide on promotions, job extensions, or job rotations. During the COVID19 crisis, many organizations discovered that employees were capable of more things than management had imagined. Overlooking potential sources of recruits has always been common, but COVID19 magnified its effects. Keep in mind that while we have used LMM in a broad sense in this work, it is primarily about not seeing a potential source as opposed to biases or stereotypes. Biases and stereotypes may lead to LMM, but they are not the only sources of LMM.

When afflicted by LMM, an organization may overlook a potential source of qualified employees by failing to advertise in channels that lead to these candidates, for example. This oversight may be governed by stereotype or bias. It may also simply be a result of 'this is the way we've always done it,' a failure to adapt. Another way of excluding potential viable candidates is by advertising above the job-seeking candidates with more qualifications than the job requires. This may mean that qualified candidates eliminate themselves from the candidate pool.

Weick (1993) identified four sources of resilience that lead to better sensemaking. All four depend on people who develop better understanding of their environment. When LMM intervenes, the organization becomes less resilient to crises, but also less resilient in support of sensemaking.

Proposition 6: Resilient organizations adapt to technological changes that help them and their employees maintain organizational continuity.

In the context of the COVID19 crisis, the most obvious failure of organizations to embrace technologies is ignoring employee preferences for remote work. Some workers have become more efficient and more effective as remote workers than as in-person workers. Some benefit from lower childcare costs and less stress in worrying about the children, the commute, and the potential negative aspects of office politics. These latter are more likely in traditional settings than in remote settings. In denying some workers the choice of remote work, some organizations are sacrificing access to important parts of the labor market.

Resilience in this sense ties back to organizational sensemaking and the degree to which LMM can disrupt that process. It directly affects the maps or cues that are retained. Organizations are nest of plausible identities. If remote work or other technologies fit the organization's identities poorly, then the organization is likely to suffer in the context of contested labor markets and factor market rivalry.

We see versions of technology embraced throughout the hospitality industry. It may not be immediately apparent, but home delivery of restaurant food, while hardly new, was hardly common to many restaurants that have now adopted it wholeheartedly. The technology for home delivery-someone's car-was hardly new, but the level of use and the widespread adoption of the process were new, and products of the pandemic. This is adopting new technologies in the same way that Walmart and others have adopted selfcheckout. Self-checkout is another embrace of technology with HRM implications, also accelerated by the pandemic. 


\section{DISCUSSION}

These propositions underscore the importance of organizational sensemaking in a crisis, but also underscore the importance of overcoming disruptions to that sensemaking. LMM disrupts both external and internal aspects of sensemaking, so it must be addressed through HRM strategies and tactics. As the TDOR model suggests, it is not sufficient to respond only after the crisis has had its negative impacts. While agility will help deal with immediate problems, resilience has a recursive relationship with sensemaking that brings it to the forefront in helping lean organizations adapt to crises led by labor market issues. The pandemic was a crisis by itself in the early stages as organizations, including institutions like national governments were trying to deal with the 'inconceivable,' but the labor crisis is lingering. It will require more study and constant process of revision. In fact, it will require the research community to engage in a broad process of sensemaking.

\section{AREAS FOR FUTURE RESEARCH}

This arena offers great potential for future research. First, the pandemic will have lingering effects on the labor market, so those effects will need to be parsed and organized within the context of sensemaking and HRM strategy. Second, each of the propositions that we have posed will need to be tested both qualitatively and quantitatively. Third, the adjustments made by organizations to this pandemic need to be codified to help prepare for the next crisis, whatever its nature. Finally, case studies documenting organizations who best prepare for pandemics and other disruptions using the propositions and guidelines

outlined in this research are needed along with teaching notes for business faculty and students within higher education. Preparing the next generation of business leaders to be more prepared will lessen the impacts and disruptions of future pandemics.

\section{REFERENCES}

Agrawal, S., De Smet, A., Lacroix, S., \& Reich, A. (2020). To emerge stronger from the COVID-19 crisis, companies should start reskilling their workforces now. McKinsey Insights.

Akkermans, J., Seibert, S.E., \& Mol, S.T. (2018). Tales of the unexpected: Integrating career shocks in the contemporary career's literature. SA Journal of Industrial Psychology, 44(1), 1-10.

Ancona, D. (2012). Sensemaking: Framing and Acting in the Unknown. In S. Snook, N. Nohria, \& R. Khurana (Eds.), The handbook for teaching leadership: Knowing, doing, and being (pp. 3-19). Sage.

Arlotta, C.J. (2018, September 7). How Cross-Training Programs Benefit Hoteliers. Hotel Business. Retrieved from https://www.hotelbusiness.com/how-cross-training-programs-benefit-hoteliers/

Balmer, J.M. (2011). Corporate marketing myopia and the inexorable rise of a corporate marketing logic: Perspectives from identity-based views of the firm. European Journal of Marketing.

Caligiuri, P., De Cieri, H., Minbaeva, D., Verbeke, A., \& Zimmermann, A. (2020). International HRM insights for navigating the COVID-19 pandemic: Implications for future research and practice.

Cheung, S.Y., \& Woo, L. (2021). Age stereotypes and the job suitability of older workers from hotel managers' perspectives. International Journal of Hospitality Management, 95, 102932.

Cook, I. (2021, September 15). Who Is Driving the Great Resignation? HBR: Human Resource Management. Retrieved from https://hbr.org/2021/09/who-is-driving-the-great-resignation

Eagly, A.H., Nater, C., Miller, D.I., Kaufmann, M., \& Sczesny, S. (2020). Gender stereotypes have changed: A cross-temporal meta-analysis of US public opinion polls from 1946 to 2018. American Psychologist, 75(3), 301.

Ellram, L.M., Tate, W.L., \& Feitzinger, E.G. (2013). Factor-market rivalry and competition for supply chain resources. Journal of Supply Chain Management, 49(1), 29-46.

González, M.J., Cortina, C., \& Rodríguez, J. (2019). The role of gender stereotypes in hiring: A field experiment. European Sociological Review, 35(2), 187-204. 
Gross, A., Morphet, S., \& Lyon, L. (2020). Flex for Success: Five Practices the Build a Flexible Workforce. Bain and Company Report. Retrieved from https://www.bain.com/insights/flex-forsuccess-five-practices-that-build-a-flexible-workforce/

Hartnett, K. (2021). Steeling for the next surge. [online] Nashville Post. Retrieved from https://www.nashvillepost.com/nashville_post_magazine/steeling-for-the-nextsurge/article_1aee3c5e-e8f8-59f1-9cf9-7d736e3a95d5.html

Hillmann, J., \& Guenther, E. (2021). Organizational resilience: A valuable construct for management research? International Journal of Management Reviews, 23(1), 7-44.

Hirsch, P.B. (2021). The great discontent. Journal of Business Strategy. (online publication)

Ivanov, D. (2021). Lean resilience: AURA (Active Usage of Resilience Assets) framework for postCOVID-19 supply chain management. The International Journal of Logistics Management.

Junior, G.T., \& Saltorato, P. (2021). Workforce agility: A systematic literature review and a research agenda proposal. Innovar, 31(81).

Koerner, S., \& Staller, M.S. (2021). From Data to Knowledge: Training of Police and Military Special Operations Forces in Systemic Perspective. Special Operations Journal, 7(1), 29-42.

Kudesia, R.S. (2017). Organizational sensemaking. In Oxford research encyclopedia of psychology.

LeMay, S., Helms, M.M., Kimball, B., \& McMahon, D. (2017). Supply chain management: The elusive concept and definition. The International Journal of Logistics Management.

LeMay, S.A., \& McMahon, R.D., Jr. (1999). The Growth and Development of Logistics Personnel: Retail Warehousing. In Annual Conference Proceedings 1999.

LeMay, S.A., McMahon, D., Batchelor, J., \& Keller, S. (2020). Journal of Transportation Management, 31(1), 9-22.

Lengnick-Hall, C.A., Beck, T.E., \& Lengnick-Hall, M.L. (2011). Developing a capacity for organizational resilience through strategic human resource management. Human Resource Management Review, 21(3), 243-255.

Levitt, T. (1960). Growth and profits through planned marketing innovation. Journal of Marketing, 24(4), $1-8$.

Markman, G.D., Gianiodis, P.T., \& Buchholtz, A.K. (2009). Factor-market rivalry. Academy of Management Review, 34(3), 423-441.

Maurer, R. (2020). Study Finds Productivity Not Deterred by Shift to Remote Work. SHRM. Retrieved from https://www.shrm.org/hr-today/news/hr-news/pages/study-productivity-shift-remote-workcovid-coronavirus.aspx

Milton, G. (2016). Churchill's Ministry of Ungentlemanly Warfare: the Mavericks Who Plotted Hitler's Defeat. New York: Picador.

Miner, J.B. (2006). Organizational behavior three: Historical origins, theoretical foundations, and the future (Vol. 3). ME Sharpe.

Mullen, C. (2021). Covid has made performance evaluations simpler, more empathetic. Bizwomen, The Business Journals.

Opengart, R., Ralston, P.M., \& LeMay, S. (2018). Labor markets: Preventing rivalry and myopia through HRM. Journal of Organizational Effectiveness: People and Performance.

Pinker, S. (2021). Rationality: What It Is, Why It Seems Scarce, Why It Matters. Penguin.

Radzius, R., Pang, K., \& Gandy, S. (2014, March). 1st SFG (A) Operational Cycle: The Continuous Execution of FID and UW. Special Warfare, pp. 30-33.

Ralston, P., LeMay, S., \& Opengart, R. (2017). Factor market myopia: A driver of factor market rivalry. Transportation Journal, 56(2), 167-183.

Rosalsky, G. (2021, October 19). Why are so many Americans Quitting Their Jobs? NPR: Planet Money. Retrieved from https://www.npr.org/sections/money/2021/10/19/1047032996/why-are-so-manyamericans-quitting-their-jobs

Tang, S., Zhang, X., Cryan, J., Metzger, M.J., Zheng, H., \& Zhao, B.Y. (2017). Gender bias in the job market: A longitudinal analysis. Proceedings of the ACM on Human-Computer Interaction, l(CSCW), 1-19. 
Tekin, E., Hopp, W.J., \& Van Oyen, M.P. (2002). Benefits of skill chaining in production lines with cross-trained workers. Manufacturing \& Service Operations Management, 4(1), 17-20.

Van Epps, G. (2008). Relooking unit cohesion: A sensemaking approach. Military Review, 88(6), 102110.

Walter, A.T. (2021). Organizational agility: ill-defined and somewhat confusing? A systematic literature review and conceptualization. Management Review Quarterly, 71(2), 343-391.

Wegmann, R.M. (2020). A grounded theory for the performance of temporary disaster response teams. Journal of Organizational Effectiveness: People and Performance.

Weick, K.E. (1988). Enacted sensemaking in crisis situations [1]. Journal of Management Studies, 25(4), $305-317$.

Weick, K.E. (1993). The collapse of sensemaking in organizations: The Mann Gulch disaster. Administrative Science Quarterly, pp. 628-652.

Weick, K.E. (1995). Sensemaking in organizations (Vol. 3). Sage.

Weick, K.E. (2003). Theory and practice in the real world. In The Oxford handbook of organization theory.

Weick, K.E., Sutcliffe, K.M., \& Obstfeld, D. (2005). Organizing and the process of sensemaking. Organization Science, 16(4), 409-421.

Wilmot, M.P., Wanberg, C.R., Kammeyer-Mueller, J.D., \& Ones, D.S. (2019). Extraversion advantages at work: A quantitative review and synthesis of the meta-analytic evidence. Journal of Applied Psychology, 104(12), 1447.

Wu, A.H. (2017). Gender stereotyping in academia: Evidence from economics job market rumors forum. Unpublished manuscript.

Wu, A.H. (2018, May). Gendered language on the economics job market rumors forum. In AEA Papers and Proceedings (Vol. 108, pp. 175-79).

Zahra, S.A., \& Chaples, S.S. (1993). Blind Spots in Competitive Analysis. The Academy of Management Executive (1993-2005), 7(2), 7-28. Retrieved from http://www.jstor.org/stable/4165119 